\title{
GFOREETI
}

\section{El crowdfunding en el Perú. Cronología de una regulación pendiente}

\begin{abstract}
Annie Collazos *
Resumen. - Teniendo en cuenta la importancia que día a día vienen adquiriendo los mecanismos de financiamiento alternativo que se brindan a través de plataformas digitales y los beneficios que vienen generándose para nuevos emprendimientos, resulta relevante revisar los principales argumentos que justificarían una regulación específica de este tipo de servicios financieros, así como la normativa vigente que podría aplicársele a este tipo de operaciones.

Abstract. - Bearing in mind the importance that alternative financing mechanisms provided through digital platforms are currently acquiring as well as the benefits that are being generated for new entrepreneurships, it is important to review the main arguments that would justify a specific regulation of this type of financial services, as well as the current regulations that could be applied to this type of operations.
\end{abstract}

* Abogada por la Universidad Nacional Mayor de San Marcos. Asociada del estudio Philippi, Prietocarrizosa, Ferrero y DU \&Uría. Las opiniones vertidas en este artículo son de exclusiva responsabilidad de la autora. 


\section{Introducción}

Desde hace varios años atrás es ampliamente conocido que la tecnología está presente en diferentes sectores de la industria y que sus avances no solo implican la creación de robots o de casas inteligentes, sino de cambios en nuestro estilo de vida tan cotidianos como la forma en la que ahora solicitamos un taxi, cómo alquilamos departamentos cuando viajamos fuera del país o del departamento y hasta cómo compramos toda clase de productos importados.

Lo que ha logrado la tecnología a través de la creación de las plataformas digitales antes mencionadas es una nueva forma de interacción entre la oferta y la demanda en tiempo real y sin necesidad de coincidir en el mismo espacio geográfico. Conectar a las personas que tienen una necesidad (un taxi, un alojamiento, un producto) con las personas que pueden satisfacerla de una manera fácil y rápida.

Si bien es cierto que la oferta y la demanda han existido desde hace mucho tiempo atrás y en casi todos los países del mundo existe normativa al respecto, la tecnología y la globalización han generado que las reglas de juego de esta común interacción cambien día a día.

Los avances tecnológicos no han sido ajenos al sector financiero. Día con día los bancos actualizan sus aplicaciones para facilitar nuestras operaciones. Lo que antes implicaba largas colas en una institución bancaria, ahora se puede lograr desde cualquiera de nuestros celulares y en un par de minutos. Sin embargo, la tecnología no solo ha facilitado que las instituciones bancarias y financieras mejoren sus servicios, sino que también ha permitido el ingreso de nuevos actores, una especie de "outsiders" que, a pesar de no ser agentes tradicionales del sector financiero, se presentan como una fuerte competencia a éstos, al ofrecer servicios financieros más flexibles, menos costosos y mucho más ágiles. A estos outsiders se les conoce como fintech.

\section{Las fintech en el Perú}

Como se señala en múltiples foros, el término fintech hace alusión a la combinación de dos palabras en inglés: Finance and Technology. Sin embargo, para efectos de este artículo al utilizar el término fintech nos estaremos refiriendo a toda "empresa orientada a crear e implementar innovaciones tecnológicas para proveer productos y servicios financieros específicos, muchas veces de una forma más eficiente y a menor costo que las tradicionales empresas del sistema financiero"1.

Como es reconocido por el propio Banco Central de Reserva del Perú (en adelante, el "BCRP") el desarrollo de las fintech en nuestro país se ha visto favorecido por el creciente nivel de accesibilidad al internet, el bajo costo de la tecnología y por un

1 Banco Central de Reserva del Perú (BCRP). Fintech en Perú. Reporte de Estabilidad Financiera, Noviembre 2017, p. 79.

2 Ídem. 
contexto socioeconómico que aún no logramos superar como país: la inclusión social y la informalidad, que también se manifiestan en el sector financiero.

Para finales del año 2017 la presencia de las fintech en nuestro país era la siguiente:

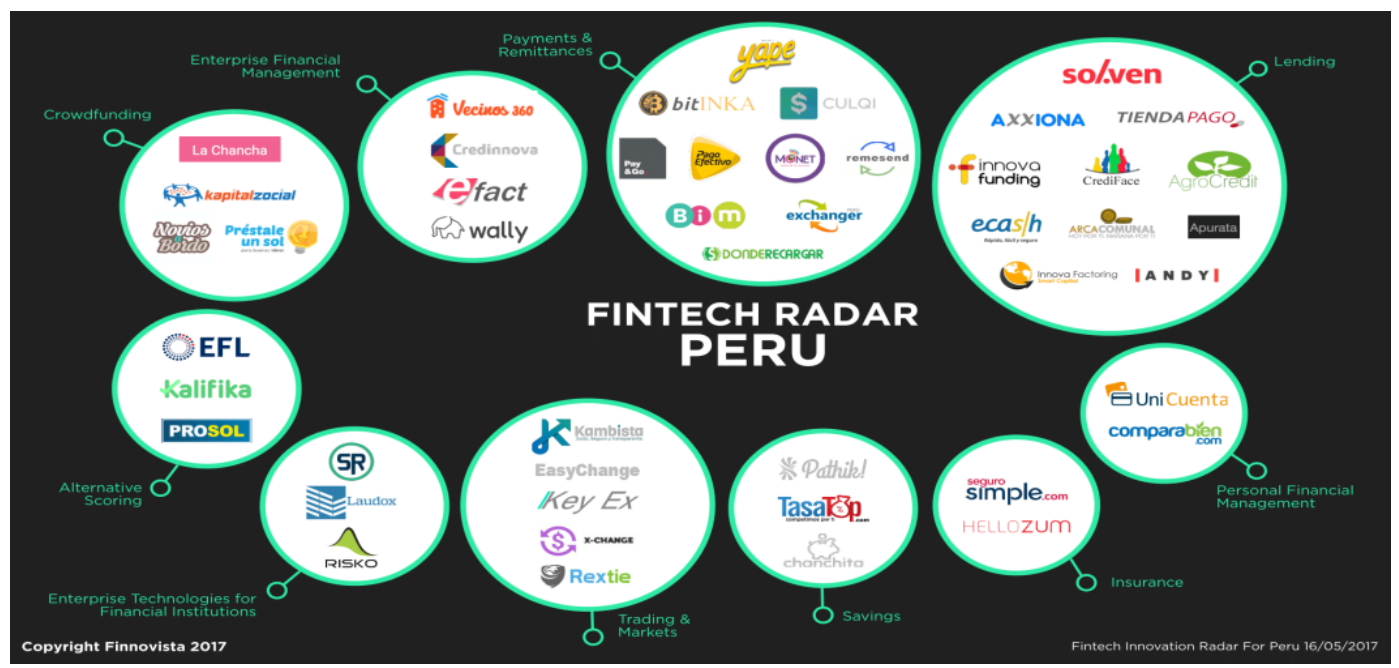

Fuente: Finnovista ${ }^{3}$

En mayo del 2017 se llevó a cabo el primer Lima Fintech Forum, evento que permitió que se pusiera sobre la mesa las diferentes perspectivas que los partícipes y supervisores del sistema financiero tenían respecto a las fintech en miras a una futura regulación.

Es así que, en noviembre de ese mismo año, en el marco del Business Innovations Summit 2017, en el cual el Superintendente Adjunto de Riesgos de la Superintendencia de Banca y Seguros y AFP (“SBS”), Alejandro Medina, anunció que su institución estaba trabajando en la elaboración de un proyecto de ley sobre "financiamiento alternativo", el cual incluiría la modalidad de crowdfunding, definiendo a los mismos como servicios financieros de préstamos a través de plataformas tecnológicas ${ }^{4}$.

Si bien no hemos encontrado la propuesta normativa a la que se hace referencia, sí hemos tenido acceso a una presentación ${ }^{5}$ elaborada por el citado Superintendente Adjunto, que habría sido utilizada en el Lima Fintech Forum del 2018 y de la cual se desprende que su propuesta de marco normativo se desarrollaría de manera selectiva y focalizada (pues no todas las actividades que realiza una plataforma

3 Finnovista (2017). Fintech Radar Perú: 47 Startups Fintech impulsan la oferta de mejores servicios financieros en Perú.. Consultado el 12 de octubre del 2018, Finovisa. https://www.finnovista.com/fintech-radar-peru-45-startups/

4 SÁNCHEZ MARTÍNEZ, César. "La SBS trabaja en un proyecto de ley sobre financiamiento alternativo a través del crowdfunding". Consultado el 8 de octubre de 2018 en Microfinanzas. https:/ / www.microfinanzas.pe/2017/index.php/2017/noviembre/400-la-sbs-trabaja-en-unproyecto-de-ley-sobre-financiamiento-alternativo-a-traves-del-crowdfunding.

5 Superintendencia de Banca, Seguros y AFP (2018) "Fintech y consideraciones para su regulación" $\begin{array}{llllll}\text { Consultado } & \text { el } & 08 & \text { de } & \text { octubre } & \text { de } \\ 2018 & \text { en }\end{array}$ http://www.sbs.gob.pe/Portals/0/jer/SUPERVISION_OP1/LimaFintechForum_SBS_Mayo_2 018.pdf. 
digital tienen carácter financiero) e implicaría una vigilancia periódica.

Por su parte, Carlos Rivero, Superintendente Adjunto de Investigación y Desarrollo de la Superintendencia de Mercado de Valores ("SMV"), durante el desarrollo del Lima Fintech Forum del presente año informó que la SMV propondría una ley para regular a las fintech dedicadas al crowdfunding.

Para Magali Silva7, asesora de Gerencia General del BCRP, un eventual marco regulatorio de las fintech debería incluir, entre otros, los siguientes principios:

a) Proporcionalidad regulatoria (no establecer barreras más altas que a las empresas financieras existentes);

b) Una visión integral del mercado, que tenga en cuenta la multisectorialidad de las empresas fintech;

c) Una política de integración regional, para aprovechar bloques como el de la Alianza del Pacífico, y, sobre todo, brindar a los inversionistas la certeza jurídica necesaria para el desarrollo del sector.

Por su parte, Marylyn Choy ${ }^{8}$, Gerente Central de Operaciones del BCRP, considera que, en el marco de la regulación pendiente, debería contemplarse "la validez legal para los instrumentos emitidos, la seguridad de la información, protección de datos, lavado de dinero, estándares para el reporte a las autoridades, validez de los contratos inteligentes, jurisdicción para la aplicación de las normas de acuerdo con la ubicación geográfica, etc." Es importante precisar que muchos de los aspectos antes mencionados podrían ya ser atendidos con normativa ya existente.

Teniendo en cuenta las propuestas de los funcionarios de la SBS, SMV y del BCRP se desprende su intención de lograr una regulación específica para los outsiders; tendencia que es compartida por otros países como Estados Unidos, Italia, España y recientemente México, que desde marzo del presente año cuenta con la Ley para regular las Instituciones de Tecnología Financiera9.

Sin embargo, ello no debería ser el único enfoque regulatorio por considerar,

6 LEÓN ARGE, Nicol. "SMV propondrá una ley para regular el Crowdfunding”. Consultado el 8 de octubre del 2018 en http://masfinanzas.com.pe/fintech/smv-propondra-una-ley-pararegular-el-crowdfunding/

7 SILVA VELARDE-ÁLVAREZ, Magali. El acelerado crecimiento de las fintech y los desafíos para su regulación. Lima, Moneda, 2017, № 171, pág. 46.

8 CHOY CHONG, Marylin. Las Fintech y los Bancos Centrales. Lima, Moneda, 2018, No 174, pág. 46.

9 El 9 de marzo del presente año se publicó en el Diario Oficial de la Federación el Decreto que aprueba la Ley para regular las Instituciones de Tecnología Financiera, de forman complementaria a la referida Ley se aprobó reformar y adicionar diversas disposiciones de la ley de instituciones de crédito, de la ley del mercado de valores, de la ley general de organizaciones y actividades auxiliares del crédito, de la ley para la transparencia y ordenamiento de los servicios financieros, de la ley para regular las sociedades de información crediticia, de la ley de protección y defensa al usuario de servicios financieros, de la ley para regular las agrupaciones financieras, de la ley de la comisión nacional bancaria y de valores y, de la ley federal para la prevención e identificación de operaciones con recursos de procedencia ilícita. Diario Oficial de la Federación. Ciudad de México, 9 de marzo de 2018. Consultado el 8 de octubre del 2018 en http:/ / www.dof.gob.mx/nota_detalle.php?codigo=5515623\&fecha=09/03/2018 


\section{El Crowdfunding en el Perú \\ Cronología de una regulación pendiente}

teniendo en cuenta que también existe la posibilidad de potenciar la normativa vigente que resultaría aplicable a los outsiders en función a la actividad y/o servicio que desarrollen.

\section{El crowdfunding y sus modalidades}

El crowdfunding, o financiamiento colectivo, es uno de los servicios más populares que las fintech ofrecen. Es así como la tecnología ha permitido que se generen plataformas digitales que, entre otras cosas, puedan ofrecer una modalidad de financiamiento alternativo que desde hace varios años atrás ha ido tomando fuerza, hasta el punto de ser ahora objeto de análisis por legisladores de diversas jurisdicciones.

Consideramos relevante identificar que el crowdfunding requiere de ciertos elementos básicos: (i) una plataforma digital, (ii) un administrador de la referida plataforma, (iii) el titular del proyecto que se desea financiar y (iv) las personas que entregarán su dinero a través de esa plataforma, a quienes en este artículo denominaremos como "patrocinadores".

En el caso peruano, tenemos que desde el año $2013^{10}$ ya se hacía eco en los diarios de experiencias foráneas del crowdfunding y a las modalidades bajo las cuales éste se venía desarrollando pudiendo identificarse cuatro categorías en función a las finalidades y/o expectativas de los patrocinadores.

Hoy en día si se ingresa a una de las plataformas que realizan crowdfunding, se podría estar frente a cualquiera de los siguientes escenarios:

a) Que para el financiamiento del proyecto se solicite la entrega de sumas de dinero (sin establecer un monto mínimo) sin la obligación de otorgar algún beneficio a favor del patrocinador.

El caso anterior se subsume bajo la figura ya existente de la donación. Bajo esta modalidad, los partícipes acceden a entregar sumas dinerarias a título gratuito sin tener derecho a recibir algún tipo de beneficio por dicha entrega. La campaña de recaudamiento puede comprender la entrega de recompensas (regalos simbólicos) por los aportes realizados, como muestra de gratitud.

Una plataforma muy conocida que utiliza esta modalidad es Kickstarter.

b) Que el proyecto que requiere el financiamiento consista en la elaboración y/o manufactura de determinados productos y que, como consecuencia de ello, la campaña de recaudamiento ofrezca la entrega de dichos productos a cambio un determinado monto de dinero aportado.

10 Diario Gestión.¿Es el 'crowdfunding' una buena alternativa de financiación?". Consultado el 8 de octubre del 2018 en https:/ / gestion.pe/tendencias/management-empleo/crowdfunding-buenaalternativa-financiacion-55858 
Como lo explica claramente, Morachimo11, el escenario anterior implica la compra de un bien futuro. El producto aún no existe y para que exista se necesita del financiamiento, motivo por el cual el patrocinador entrega su dinero y el titular del proyecto, una vez obtenido el financiamiento total requerido, estará obligado a entregar productos a los patrocinadores.

Resulta importante resaltar que las plataformas que ofrecen esta modalidad de crowdfunding, como Kickstarter, precisan que el titular del proyecto no recibirá ninguna suma dineraria hasta que se haya alcanzado el monto del financiamiento requerido. De no alcanzarse, el dinero entregado a través de la plataforma será devuelto a los patrocinadores.

c) Quien requiere el financiamiento a través de la plataforma se obliga a devolver el dinero que le sea entregado en un determinado periodo de tiempo y sujeto a la generación de intereses. Este supuesto encaja en la definición de préstamo, motivo por el cual esta sería una de las modalidades que tanto local como internacionalmente se consideran como crowdfunding financiero.

d) Que en la campaña de recaudación se solicite a los patrocinadores que inviertan en un determinado proyecto, ofreciéndoles a cambio de acciones o participaciones representativas del capital social de la sociedad titular del proyecto. Esta modalidad califica como una oferta de valores mobiliarios, y, teniendo en cuenta que es abierta al público, se consideraría una oferta pública. Esta modalidad también está incluida dentro de la categoría de crowdfunding financiero.

Teniendo en cuenta la descripción de las modalidades de crowdfunding antes señaladas, resulta evidente que algunas de ellas podrían estar transgrediendo algunas normas vigentes de nuestro país, especialmente las referentes al otorgamiento de préstamos (como servicio financiero) y a las ofertas públicas de acciones (en el marco del mercado de valores).

Por dicho motivo, en enero del 201412, la SMV emitió un comunicado mediante el cual se enfatizó que toda invitación que una persona natural o jurídica dirija al público en general para realizar cualquier acto referido a la colocación o venta de valores mobiliarios es una oferta pública de valores, por lo que debe observar el régimen legal establecido y ser objeto de supervisión de la SMV.

\section{Legislación aplicable}

Como lo hemos señalado en las secciones anteriores, desde hace años atrás se viene

11 MORACHIMO RODRÍGUEZ, Miguel, Director de la ONG Hiperderecho, Lima, 2013. Entrevista publicada a través de la revista Derecho \& Sociedad Num. 41, http:// revistas.pucp.edu.pe/index.php/derechoysociedad/article/view/12772

12 Diario Gestión (2014) "El crowdfunding a través de la emisión de valores o aportes de capital no están autorizados, advierte la SMV". Consultado el 8 de octubre del 2018 en https://gestion.pe/economia/mercados/crowdfunding-traves-emision-valores-aportescapital-autorizados-advierte-smv-1705 
trabajando en la idea de una regulación específica para el crowdfunding y otros servicios de las fintech; sin embargo, ello no debe interpretarse como si estas plataformas operaran sin regulación alguna, toda vez que el desarrollo de sus actividades comprende diversos aspectos que han sido regulados desde hace años atrás.

En línea con lo anterior y teniendo en cuenta las más usuales preocupaciones de los patrocinadores que participan en las plataformas de crowdfunding, podríamos sintetizar la normativa aplicable en los siguientes puntos:

1) Las disposiciones del Código de Protección y Defensa del Consumidor (Ley 29571) y sus normas complementarias, en caso como los de información inexacta o errónea y/o falta de idoneidad en el servicio.

2) Las disposiciones de la Ley de Protección de Datos Personales (Ley 29733) y sus normas complementarias. Uso indebido de los datos personales que los patrocinadores proporcionen a las plataformas.

Asimismo, en lo que se refiere a los usos y efectos que el crowdfunding podría tener y que afectarían a los agentes tradicionales e instituciones del sistema financiero, la normativa aplicable sería la siguiente:

3) Uso indebido de las plataformas para transferir dinero de origen ilícito. Resultarán aplicables las disposiciones del Decreto Legislativo de lucha eficaz contra el lavado de activos y otros delitos relacionados a la minería ilegal y crimen organizado (Decreto Legislativo 1106) y sus normas complementarias.

4) Ofertas públicas que se realizan a través de las plataformas digitales y que tengan como finalidad captar inversionistas a cambio de acciones y/o participaciones en el capital social de las empresas titulares de los proyectos que requieren financiamiento. Deberán sujetarse al cumplimiento de las disposiciones del Texto Único Ordenado de la Ley del Mercado de Valores (Decreto Supremo 093-2002-EF) toda vez que de conformidad con el artículo 56 de la Ley General de Sociedades (Ley 26887) cuando la oferta a terceros tenga la condición legal de oferta pública, le es aplicable la legislación especial.

5) Operaciones de préstamos y/o depósitos sujetos a rendimiento que se realicen mediante las referidas plataformas digitales. Estarán sujetas a las limitaciones establecidas en Ley General del Sistema Financiero y del Sistema de Seguros y Orgánica de la Superintendencia de Banca y Seguros - Ley 26702 (en adelante, la "Ley del Sistema Financiero").

Como se precisa en el artículo 11 de la Ley del Sistema Financiero se establece expresamente que hay ciertas actividades que requieran la autorización de la SBS para ser realizadas. Entre dichas actividades se encuentra la de "dedicarse al giro propio de las empresas del sistema financiero, y en especial, a captar o recibir en forma habitual dinero de terceros, en depósito, mutuo o cualquier otra forma, y colocar habitualmente tales recursos en forma de créditos, 
inversión o de habilitación de fondos, bajo cualquier modalidad contractual".

6) Funcionalidad del sistema de pago utilizado por la plataforma digital. De conformidad con la Ley de los Sistemas de Pagos y de Liquidación de Valores (Ley 29440) el BCRP está facultado para requerir información al respecto.

Como precisa el BCRP:

“En el caso de la Ley de los Sistemas de Pago y de Liquidación de Valores, las fintech de pagos son consideradas como proveedores de servicios de pago, por lo que el BCRP les puede solicitar información para monitorear su progreso. Al respecto, dicha Ley señala que el BCRP puede requerir a los proveedores de servicios de pagos, información que le permita conocer la naturaleza y volumen de sus operaciones, su funcionalidad y las medidas de control de los riesgos"13.

\section{Conclusiones}

Como hemos expuesto a lo largo del presente artículo, los funcionarios de las principales instituciones de nuestro país comparten la misma tendencia regulatoria respecto a las fintech: regulación específica. Para ellos estaría pendiente la aprobación de nuevas normas con aplicación específica para los servicios financieros que se brinden a través de las fintech.

Sin embargo, consideramos importante evaluar un enfoque regulatorio distinto, que implique: (i) trabajar con la normativa vigente que a la fecha puede ser aplicada también a las fintech, como a cualquier otro sujeto de derechos, (ii) ratificar y potenciar las potestades de supervisión de los entes competentes y (iii) regular únicamente en los supuestos en los que sea estrictamente necesario, a fin de no afectar ni desincentivar el desenvolvimiento del fenómeno fintech en nuestro país.

13 Banco Central de Reserva del Perú (BCRP). Fintech en Perú. Reporte de Estabilidad Financiera, Noviembre 2017, p. 80 . 